\title{
Research on network technology of emergency communication based on Ad Hoc network
}

\author{
Xiao Zhang ${ }^{1, a}$, Hai-Yan Zhao ${ }^{1, b}$, Ming-shun Xing ${ }^{1, c}$ \\ ${ }^{1}$ Xi'an Communication Institute, Xi'an 710106, China \\ a51375908@qq.com, b813086903@qq.com, ${ }^{\circ} 583822272 @ q q . c o m$
}

Key words: emergency communication; wireless ad hoc networks; hierarchical framework;

Abstract: Traditional emergency communication system cannot meet the status of emergency communications needs. In accordance with the requirements of the establishment of a reliable, flexible and robust emergency communication network, this paper introduces in detail the emergency communication network, the design idea and key technology based on the ad hoc network.

Emergency communication can effectively deal with major natural disasters and emergencies by providing timely and reliable communication service and it has special requirements on communication and distinctive features [1]: communication services highly survivable and strong adaptability, communication time of emergency and location with the meaning of uncertainty and information diversity and process of transient. The existing communication system has not fully considered the requirements and characteristics of the communication.

At present, the domestic emergency communication technology is relatively backward, and the self-organized ability and survivability of the emergency communication system is low. When the communication infrastructure is not available, emergency communications support needs to be provided with the help of satellite, shortwave and trunking communication system. But it is still difficult to meet the rapid formation of reliable, efficient, robust adaptation requirements of emergency communication network of various types of user groups. As the public communication network is not reliable, and easy to cause network congestion; trunking communication system can set up fast call and support group call, broadcast call and supplementary services, but its coverage and communication capacity is small, usually confined to command and control application, and still rely on the basic communication facilities; satellite communications is vigorous and healthy, covering a wide range, but it has limited transmission capacity, high cost in deployment and difficulty in technical support. Therefore, it is necessary to introduce new techniques and methods, deploy emergency communication network rapidly, provide diversified timely manner for all types of users to meet their demand for communication services, conduct real-time monitoring of specific areas and make rapid response to emergencies, and coordinate all rescue forces after the implementation of emergency rescue and disaster relief and post disaster reconstruction.

With the rapid development of information and network technology, many new techniques gradually mature and show the characteristic in the field of emergency communication, especially the extensive research of industry wireless network (wireless self organizing network and WSON). WSON is the organic combination of the mobile communication network and computer network, which has the characteristics of no infrastructure support, automatic network, multi hop relaying etc. It has stronger network survivability and flexibility, especially suitable for battlefield, remote areas, emergency and temporary communication occasions. The earliest wireless ad hoc networks can be traced back to the packet radio network developed by the american army in the seventies of the 20 th century [2], then gradually emerged in the form of ad hoc networks [3], wireless sensor network [4] and wireless mesh network [5], these networks have their own characteristics and applications. Such as the hybrid network can be combined with ad hoc and cellular network on the scene emergency to improve the capacity of the communication system, expand the coverage and improve the quality of service; the network and wireless sensor networks also can be deployed in the bank, for the acquisition and monitoring of specific area in real time; emergency communication system can also be deployed based on wireless mesh network of the city. 


\section{design ideas of emergency communication network based on wireless ad hoc network}

Emergency communication networks often need to operate in complex and harsh environment, the geographical and climatic conditions are varied, the network size can be either large and small. This network has the following salient features: involving a variety of network system with different abilities and characteristics, such as cable transmission network, wireless sensor network, cellular mobile communication network, the air relay system and satellite networks; contains a large number of mobile speed and the ability to deal with different communication unit and communication unit, needs communication and coordination; a wide range of business, with asymmetric information transmission characteristics, such as information received by rescue workers at the scene are more than sent ones.

According to the characteristics of emergency communication network, wireless network design is based on the actual demand for emergency communication scene, relying on the actual need of mobile wireless communication system. The fast automatic networking technology, high flexibility and strong survivability is fully developed based on the existing network infrastructure and the temporary deployment of wireless ad hoc network. The basic construction the network facilities and network without infrastructure is constructed. Wired, wireless and satellite integrated various communication means of emergency communication network are integrated to provide a flexible and reliable communication, with high survivability support platform for emergency disposal, making it possible for all kinds of personnel within the site to reach the command center timely, accurately and smoothly.

\section{design scheme of emergency communication network based on wireless ad hoc network}

In order to build a flexible, robust, scalable and versatile emergency communication network, a general heterogeneous emergency communication network hierarchical framework based on network coverage and network layering is designed, as shown in figure 1.

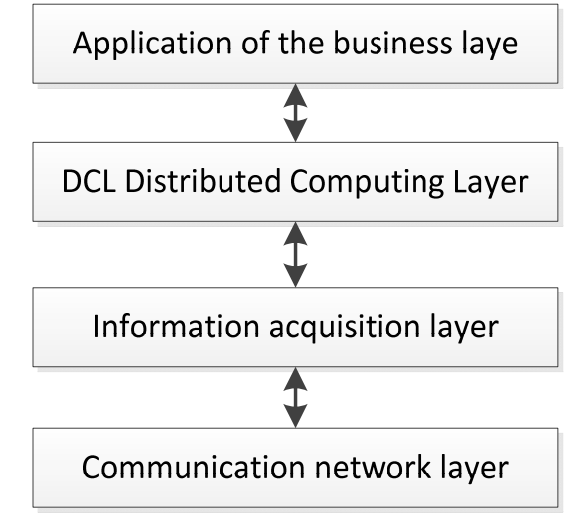

Fig.1 Layering framework of emergency communication network

The framework is divided into communication network layer, information collection layer, computing layer and application layer. Network communication layer consists of infrastructure communication network and the temporary deployment of no infrastructure communication network, providing support for the realization of three above function; information collection layer is responsible for the necessary information collection, screening and storage layer of various communication networks, and transfer the necessary management control information to the upper and lower layers; distributed computing layer mainly complete the information aggregation and intelligent information processing, the implementation of distributed computing, deep data mining and decision making; application service layer provides a variety of emergency communication applications and services.

On the basis of the hierarchical framework, design system and network architecture. From the view of system structure, heterogeneous emergency communication system is a multitier distributed 
information system, includes four layers, from bottom to top in order: network infrastructure layer, common services layer, specific functional layer and application layer. The system structure of emergency communication network is shown in Figure 2.

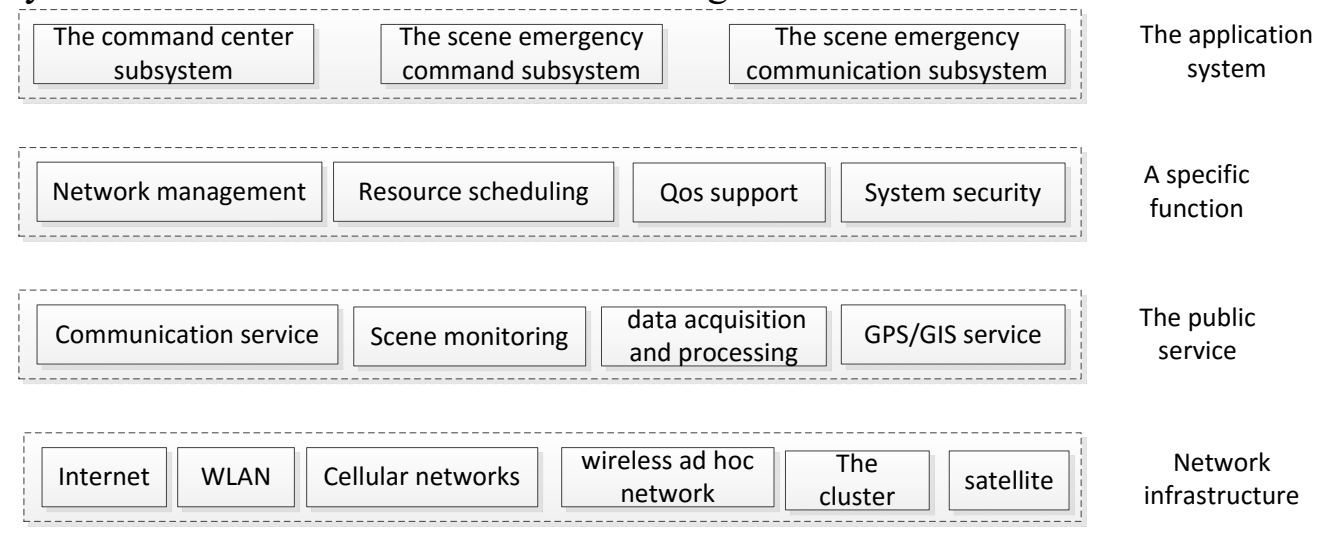

Fig.2 System structure of emergency communication network

(1) network infrastructure layer covers communications infrastructure of public communication network, such as the Internet and cellular network and private communication network (such as clusters ) and satellite communication network and wireless ad hoc networks, such as ad hoc networks and wireless sensor network infrastructure.

(2)Public service layer includes communication services, scene monitoring, data acquisition and processing and the global positioning system (global positioningsystem, GPS), and geographical information system, geographical information system, GIS) service, providing supporting service for the above function module layer. Among them, the communication service is the core service, based on underlying the deployment of various communication networks to the upper applications provide communication services (including all kinds of communication between the personnel in the emergency field and the rear command center of communication) and shield the underlying complex heterogeneous network networking details to upper application; scene monitoring to emergency network environment were real-time monitoring, data acquisition and processing will complete the underlying network information collection, storage and processing; GPS is used for positioning terminal assisted, GIS to visualize the scene emergency situation.

(3) The specific functional layer is based on the public service layer, in order to meet the needs of different applications, including network management, resource scheduling, quality of service QoS (quality of service, QoS support and system security function. The purpose is to enhance the emergency communication system of adaptability and usability. The network management involes the adjustment of network equipment configuration, the performance of the network, mobile terminal management and fault recovery; resource scheduling is responsible for the overall distribution of all kinds of resources (bandwidth, wireless spectrum, CPU and memory, etc.); QoS support provides differentiated priority service based on urgent business degree and the identity of a user ; network security ensures the safety of information storage, transmission and use, and application of the system itself.

(4) application system layer is responsible for providing all kinds of emergency communication services, such as text, voice, and multimedia information interaction, including three application subsystem: command center subsystem, field emergency command subsystem and on-site emergency communication subsystem. Command center subsystem provide on-site emergency information for backward commanders in all kinds of information expression form (message text, graphics, audio and video), in order to obtain the comprehensive situation map, make scientific decisions, give orders to the commanders at the scene. The field emergency command subsystem supports many kinds of communication technologies, which can communicate with the rear command post, report the incident area, request access to the data resources and accept the command and control. Site emergency communication subsystem provides information collection, inquiry, sending, receiving and warning services to the rescue workers. With the help of the subsystem, rescue personnel can query the on-site environmental information, upload the 
collected sensor data, communicate and collaborate, transfer textual and visual situation information to the commander, and accept the commander's instructions and warning.

In the physical network structure, heterogeneous emergency communication network is a multilayer stereo communication network, including ground and air communications facilities, which is an integrated use of wired, wireless, low altitude platform, high-altitude satellites technique to set up the integration of heterogeneous emergency communication network, its structure as shown in Figure 3.

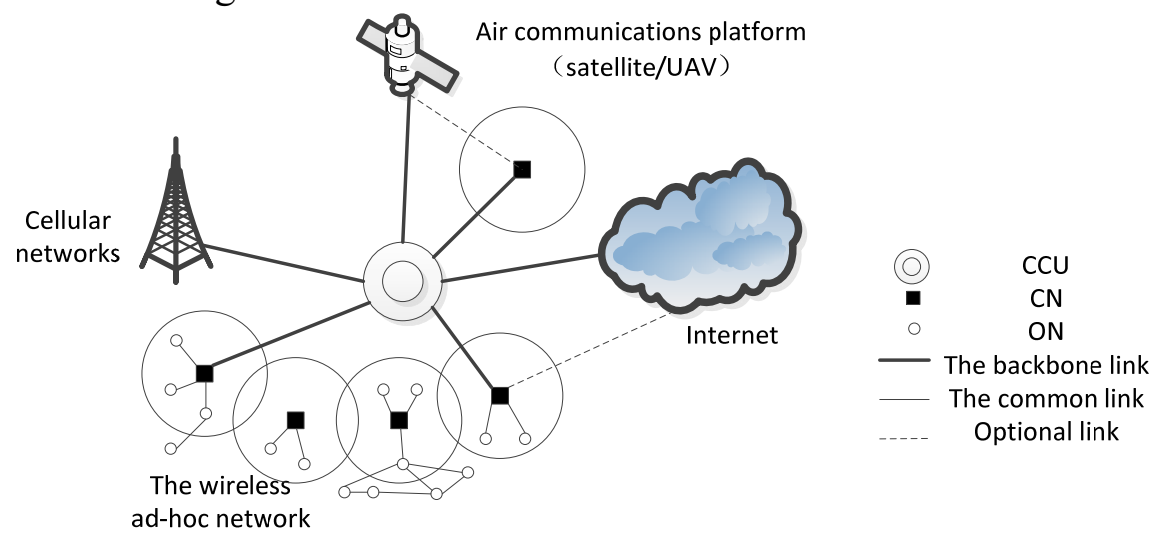

Fig.3 Network structure of integrated heterogeneous emergency communication network

\section{concluding remarks}

The quick and effective construction of emergency communication network is very important for the major natural disasters and accidents emergency rescue work. As a supplementary means in the public communication network, the construction of emergency communication network should not be limited to conventional public communication network technology, a variety of technical mean should be fully taken into consideration, especially the effective use of wireless communication technology. Through the analysis on various existing emergency field communication network technology, it can be found that the emergency field communication network should give full play to the important role of satellite communication and mobile ad hoc networks. The information obtaining and long distance transmission should be taken into consideration in the construction of emergency communication network. Of course, there are many emergency communication network technology means. For example, someone proposed to use hydrogen balloons to collect and transmit information on the accident site. In this article, the networking scheme mainly considered the comprehensive use of the communication technology, only to provide related reference for the emergency communications security department.

\section{reference}

[1] CHITI F, FANTACCI R. A broadband wireless communications system for emergency management[J]. IEEE Wireless Communications, 2008, 7(6): 8-14.

[2] LARRY S, SCOTT S. Manet gateways: radio interoperability via the internet, not the radio[J]. IEEE Communication magazine, 2008, 45(11): 51-59.

[3] ANSARI N, ZHANG C. Networking for critical conditions[J]. IEEE Wireless Communications, 2009,7(4): 73-81.

[4] TEHUANG L, WANJIUN L. On routing in multichannel wireless mesh networks: challenges and solutions[J]. IEEE Network, 2010, 22(1): 13-18.

[5] AMEER A, MOHAMED Y. A survey on clustering algorithms for wireless sensor networks[J]. Computer Communications, 2009, 30(6): 2826-2841. 